\title{
GENERALIZED CESARO MEANS OF ORDER -1
}

\author{
by I. J. MADDOX
}

(Received 18 September, 1964; and in revised form 11 January, 1965)

A series $\sum a_{n}$ is said to be summable $(C,-1)$ to $s$ if it converges to $s$ and $n a_{n}=o(1)$ [8]. It is well known that this definition is equivalent to $t_{n} \rightarrow s(n \rightarrow \infty)$, where $t_{n}=s_{n}+n a_{n}$, $s_{n}=a_{0}+\ldots+a_{n}$. The series is summable $|C,-1|$ to $s$ if the sequence $t=\left\{t_{n}\right\}$ is of bounded variation $\left(t \in\right.$ B.V.), i.e. $\sum\left|\Delta t_{n}\right|=\sum\left|t_{n}-t_{n-1}\right|<\infty$, and $\sum \Delta t_{n}=\lim t_{n}=s . \dagger \quad$ An equivalent condition is $\sum\left|a_{n}\right|<\infty, \sum a_{n}=s$ and $\sum\left|\Delta\left(n a_{n}\right)\right|<\infty$. For, suppose that $\sum a_{n}=s|C,-1|$. Since $\left\{s_{n}\right\}$ is the sequence of $(C, 1)$-means of $\left\{t_{n}\right\}$ and since $|C, 0| \subset|C, 1|$, we have $\sum\left|a_{n}\right|<\infty$ and $\sum a_{n}=s$, whence $\sum\left|\Delta\left(n a_{n}\right)\right|<\infty$. Conversely, $\sum\left|a_{n}\right|<\infty$, $\sum a_{n}=s$ and $\sum\left|\Delta\left(n a_{n}\right)\right|<\infty$ imply $t \in$ B.V. and $\sum \Delta t_{n}=s+\lim n a_{n}$. But lim $n a_{n}=0$, since $\sum\left|a_{n}\right|<\infty$.

Now let $\sum a_{n}$ be a given series, with $s_{n}=a_{0}+\ldots+a_{n}$, and define the sequence $\left\{t_{n}\right\}$ so that $s_{n}$ is the discontinuous Riesz mean of order 1 of $t_{n}$ :

$$
s_{n}=\frac{1}{\lambda_{n+1}} \sum_{k=0}^{n}\left(\lambda_{k+1}-\lambda_{k}\right) t_{k}
$$

where $0 \leqq \lambda_{0}<\lambda_{1}<\ldots<\lambda_{n} \rightarrow \infty$. Then we have

$$
t_{n}=s_{n}+\mu_{n} a_{n}, \quad \text { with } \mu_{n}=\frac{\lambda_{n}}{\lambda_{n+1}-\lambda_{n}} .
$$

We shall say that $\sum a_{n}=s\left(C, \lambda_{n},-1\right)$ if and only if $t_{n} \rightarrow s(n \rightarrow \infty)$. By the regularity of $\left(\bar{R}, \lambda_{n}, 1\right)$ summability it is easily seen that an equivalent definition is that $\sum a_{n}$ converges to $s$ and $\mu_{n} a_{n}=o(1)$. If $\lambda_{n}=n,\left(C, \lambda_{n},-1\right)$ reduces to $(C,-1)$, so that the new method generalizes the Cesarro method of order -1 .

We have used the notation $\left(C, \lambda_{n},-1\right)$ rather than $\left(\bar{R}, \lambda_{n},-1\right)$ since a definition $\ddagger$ of discontinuous $\left(\bar{R}, \lambda_{n},-1\right)$ summability is already available. Now it is known [5], that $(C, k)$ and $(\bar{R}, n, k)$ are equivalent for $-1<k<2$, and Dr Kuttner has shown me a proof, similar to that of [5], that $(\bar{R}, n,-1)$ implies $(C,-1)$ but that the converse implication is false. Thus $\left(C, \lambda_{n},-1\right)$ is not equivalent to $\left(\bar{R}, \lambda_{n},-1\right)$ even when $\lambda_{n}=n$.

Using (1) we define $\sum a_{n}=s\left|C, \lambda_{n},-1\right|$ if and only if $t \in$ B.V. and $t_{n} \rightarrow s$. Thus we have the inclusion $\left|C, \lambda_{n},-1\right| \subset\left(C, \lambda_{n},-1\right)$. We now give an equivalent condition for $\left|C, \lambda_{n},-1\right|$ summability.

THEOREM 1. $\sum a_{n}=s\left|C, \lambda_{n},-1\right|$ if and only if $\sum\left|a_{n}\right|<\infty, \sum a_{n}=s$ and $\sum\left|\Delta\left(\mu_{n} a_{n}\right)\right|<\infty$.

† All summations run from 0 to $\infty$, and we take $t_{-1}=0$.

$$
\ddagger \sum a_{n}=s\left(\bar{R}, \lambda_{n},-1\right) \text { if } \sum_{k=0}^{n}\left(1-\frac{\lambda_{k}}{\lambda_{n+1}}\right)^{-1} a_{k} \rightarrow s \quad(n \rightarrow \infty) \text {. }
$$


Proof. By the absolate regularity of $\left(\bar{R}, \lambda_{n}, 1\right), \sum a_{n}=s\left|C, \lambda_{n}-1\right|$ implies $\sum\left|a_{n}\right|<\infty$ and $\sum a_{n}=s$, whence $\sum\left|\Delta\left(\mu_{n} a_{n}\right)\right|<\infty$. Conversely, $\sum\left|a_{n}\right|<\infty, \sum a_{n}=s$ and $\sum\left|\Delta\left(\mu_{n} a_{n}\right)\right|$ $<\infty$ imply $t \in$ B.V. and $\sum \Delta t_{n}=s+\lim \mu_{n} a_{n}$. Now suppose, if possible, that $\lim \mu_{n} a_{n}=l \neq 0$. We first note that

$$
\sum_{n=1}^{\infty} \frac{1}{\mu_{n}}=\infty
$$

For the infinite product

$$
\prod_{n=1}^{\infty}\left(1+\frac{1}{\mu_{n}}\right)=\prod_{1}^{\infty} \frac{\lambda_{n+1}}{\lambda_{n}}
$$

diverges to $+\infty$, which implies that (2) holds. Since $a_{n} \sim \| / \mu_{n}$, (2) implies that $\sum a_{n}$ diverges, a contradiction. Hence $\mu_{n} a_{n}=o(1)$, so that $\sum a_{n}=s\left|C, \lambda_{n},-1\right|$.

Our next theorem gives the class of sequences $\left\{\lambda_{n}\right\}$ for which the generalized methods are equivalent to convergence (or absolute convergence). It is known [3, Theorem 21] that a sufficient condition for $\left(R, \lambda_{n}, k\right)$ summability $(k>0)$ to be equivalent to convergence is

$$
\Lambda_{n}=\frac{\lambda_{n+1}}{\lambda_{n+1}-\lambda_{n}}=O(1) .
$$

Since $\Lambda_{n}=\mu_{n}+1, \mu_{n}=O(1)$ is also a sufficient condition. Theorem 2 shows that $\mu_{n}=O(1)$ is necessary as well as sufficient for $\left(C, \lambda_{n},-1\right)$ to be equivalent to $(C, 0)$ and for $\left|C, \lambda_{n},-1\right|$ to be equivalent to $|C, 0|$, where $(C, 0)(|C, 0|)$ denotes convergence (absolute convergence).

THEOREM 2. $\left(C, \lambda_{n},-1\right)\left(\left|C, \lambda_{n},-1\right|\right)$ is equivalent to $(C, 0)(|C, 0|)$ if and only if $\mu_{n}=O(1)$, or what amounts to the same thing, if and only if $\Lambda_{n}=O(1)$.

Proof. The inclusions $\left(C, \lambda_{n},-1\right) \subset(C, 0)$ and $\left|C, \lambda_{n},-1\right| \subset|C, 0|$ follow immediately from the equivalent definitions of $\left(C, \lambda_{n},-1\right)$ and $\left|C, \lambda_{n},-1\right|$. Suppose then that $\sum a_{n}$ converges to $s$ and $\mu_{n}=O(1)$. Then $\mu_{n} a_{n}=O(1) . o(1)=o(1)$, whence $(C, 0) \subset\left(C, \lambda_{n},-1\right)$. Also it is clear that, if $\sum a_{n}=s$ implies $\mu_{n} a_{n}=o(1)$, then $\mu_{n}=O(1)$. Also it is clear that, if $\sum a_{n}=s$ implies $\mu_{n} a_{n}=o(1)$, then $\mu_{n}=O(1)$. This gives the first result of the theorem.

Now let $\sum\left|a_{n}\right|<\infty$ and $\mu_{n}=O(1)$. Then

$$
\sum\left|\Delta\left(\mu_{n} a_{n}\right)\right|=O(1) \sum\left|a_{n}\right|<\infty,
$$

so that $|C, 0| \subset\left|C, \lambda_{n},-1\right|$. Finally, suppose that $\sum\left|a_{n}\right|<\infty$ implies $\sum\left|\Delta\left(\mu_{n} a_{n}\right)\right|<\infty$, i.e. that $\sum\left|x_{n}\right|<\infty$, where

$$
x_{n}=\sum_{k=0}^{\infty} c_{n, k} a_{k},
$$

and $c_{n, n}=\mu_{n}, c_{n, n-1}=-\mu_{n-1}, c_{n, k}=0(k \neq n-1, n)$. By a theorem of Knopp and Lorentz [4], $\sum\left|x_{n}\right|<\infty$ if and only if

$$
\sup _{k} \sum_{n=0}^{\infty}\left|c_{n, k}\right|<\infty \text {. }
$$


Using the necessity of this condition in our case we see that $\mu_{n}=O(1)$. This proves the theorem.

The next result involves a change in the type of summability. We take sequences $\left\{\lambda_{n}\right\}$ and $\left\{\lambda_{n}^{\prime}\right\}$, with the corresponding sequences $\left\{\mu_{n}\right\}$ and $\left\{\mu_{n}^{\prime}\right\}$.

TheOReM 3. $\left(C, \lambda_{n},-1\right) \subset\left(C, \lambda_{n}^{\prime},-1\right)$ if and only if $\Lambda_{n}^{\prime}=O\left(\Lambda_{n}\right)$.

Proof. If $\sum a_{n}=s\left(C, \lambda_{n},-1\right)$, then $a_{n}=o(1)$ and $\mu_{n} a_{n}=o(1)$; and $\Lambda_{n}^{\prime}=O\left(\Lambda_{n}\right)$ then gives

$$
\mu_{n}^{\prime} a_{n}=\left(\Lambda_{n}^{\prime}-1\right) a_{n}=O\left(\left(\Lambda_{n}+1\right)\left|a_{n}\right|\right)=O\left(\left(\mu_{n}+2\right)\left|a_{n}\right|\right)=o(1),
$$

whence $\sum a_{n}=s\left(C, \lambda_{n}^{\prime},-1\right)$. Now suppose that $\left(C, \lambda_{n},-1\right) \subset\left(C, \lambda_{n}^{\prime},-1\right)$, i.e. that $t_{n}=s_{n}+\mu_{n} a_{n} \rightarrow s(n \rightarrow \infty)$ implies $t_{n}^{\prime}=s_{n}+\mu_{n}^{\prime} a_{n} \rightarrow s(n \rightarrow \infty)$. Then

$$
t_{n}^{\prime}=\sum_{k=0}^{n} c_{n, k} t_{k} \quad\left(c_{n, n}=\Lambda_{n}^{\prime} / \Lambda_{n}\right)
$$

converges to $s$ whenever $t_{n}$ does. By the Toeplitz theorem it is necessary that $c_{n, n}=O(1)$, i.e. that $\Lambda_{n}^{\prime}=O\left(\Lambda_{n}\right)$. This completes the proof.

With the restriction $\lambda_{n+1}=O\left(\lambda_{n}\right)$ we note that $\mu_{n}^{\prime}=O\left(\mu_{n}\right)$ is also necessary and sufficient.

Specializing $\lambda_{n}$ and $\lambda_{n}^{\prime}$ in Theorem 3 we have the inclusion $(C, \log n,-1) \subset(C, n,-1)$, which may be contrasted with a typical "second theorem of consistency" for Riesz means [3, Theorem 18], in which $(R, n, k) \subset(R, \log n, k)$ for $k>0$.

In the next theorem we give some results on summability factors for the methods $\left(C, \lambda_{n},-1\right)$ and $\left|C, \lambda_{n},-1\right|$, which extend and generalize some known theorems on Cesaro summability factors ([2], [7]). If $A, B$ are any summability methods, we use the notation $\left\{\varepsilon_{n}\right\} \in(A, B)$ to mean that the $A$-summability of $\sum a_{n}$ implies the $B$-summability of $\sum a_{n} \varepsilon_{n}$.

THEOREM 4. (a) $\left\{\varepsilon_{n}\right\} \in\left((C, k),\left|C, \lambda_{n},-1\right|\right)$ for $k \geqq-1$, if and only if

$$
\sum_{1}^{\infty} \Lambda_{n} n^{k}\left|\varepsilon_{n}\right|<\infty
$$

(b) $\left\{\varepsilon_{n}\right\} \in\left(\left(C, \lambda_{n},-1\right),\left|C, \lambda_{n},-1\right|\right)$ if and only if

$$
\sum\left|\varepsilon_{n}\right|<\infty \text {. }
$$

(c) $\left\{\varepsilon_{n}\right\} \in\left(|C, 0|,\left|C, \lambda_{n},-1\right|\right)$ if and only if

$$
\text { (i) } \mu_{n} \varepsilon_{n}=O(1) \text {, (ii) } \varepsilon_{n}=O(1) \text {. }
$$

(d) $\left\{\varepsilon_{n}\right\} \in(|C,-1|,|C, 0|)$ if and only if

$$
\sum_{n=k}^{\infty} n^{-2}\left|\varepsilon_{n}\right|=O\left(k^{-1}\right)
$$

Proof. (a) For the sufficiency, the well-known limitation theorem for the $(C, k)$-summability of $\sum a_{n}$ gives $a_{n}=o\left(n^{k}\right)$. Hence, since $1<\Lambda_{n}, \sum_{1}^{\infty}\left|a_{n} \varepsilon_{n}\right|=O(1) \sum_{1}^{\infty} n^{k}\left|\varepsilon_{n}\right|<\infty$, and since $\mu_{n}<\Lambda_{n}$,

$$
\sum_{1}^{\infty}\left|\Delta\left(\mu_{n} a_{n} \varepsilon_{n}\right)\right|=O(1) \sum_{1}^{\infty} \mu_{n} n^{k}\left|\varepsilon_{n}\right|<\infty .
$$


For the necessity, define

$$
\begin{aligned}
& r_{n}=\sum_{s=0}^{n} a_{s} \varepsilon_{s}+\mu_{n} a_{n} \varepsilon_{n}, \\
& q_{n}^{k}=\left\{\begin{array}{cc}
C_{n}^{k} & (k>-1), \\
s_{n}+n a_{n} & (k=-1),
\end{array}\right.
\end{aligned}
$$

where $C_{n}^{k}$ is the Cesàro mean of $\sum a_{n}$. If $\left\{\varepsilon_{n}\right\} \in\left((C, k),\left|C, \lambda_{n},-1\right|\right)$, then $\sum\left|\Delta r_{n}\right|<\infty$, whenever $\left\{q_{n}^{k}\right\}$ converges. Now, if $A_{0}^{\alpha}=1$,

$$
A_{n}^{\alpha}=\frac{(\alpha+1)(\alpha+2) \ldots(\alpha+n)}{n !} \quad(n \geqq 1),
$$

for real $\alpha$, then

$$
\begin{aligned}
\Delta r_{n} & =a_{n} \varepsilon_{n}+\Delta\left(\mu_{n} a_{n} \varepsilon_{n}\right) \\
& =\Lambda_{n} a_{n} \varepsilon_{n}-\mu_{n-1} a_{n-1} \varepsilon_{n-1} \\
& = \begin{cases}\Lambda_{n} \varepsilon_{n} \sum_{s=0}^{n} A_{n-s}^{-k-2} A_{s}^{k} C_{s}^{k}-\mu_{n-1} a_{n-1} \varepsilon_{n-1} & (k>-1), \\
\frac{\Lambda_{n} \varepsilon_{n}}{n+1} \sum_{s=0}^{n} q_{s}^{-1}-\ldots & (k=-1), \\
& =\sum_{s=0}^{n} a_{n, s}^{k} q_{s}^{k}\end{cases}
\end{aligned}
$$

where

$$
a_{n, n}^{k}= \begin{cases}\Lambda_{n} \varepsilon_{n} A_{n}^{k} & (k>-1), \\ \frac{\Lambda_{n} \varepsilon_{n}}{n+1} & (k=-1) .\end{cases}
$$

Hence by a slight modification of a theorem of Chow [2, Lemma 6†], $\sum\left|a_{n, n}^{k}\right|<\infty$, which is equivalent to the condition in $(a)$.

If $\lambda_{n}=n$ we find $\ddagger$ that $\left\{\varepsilon_{n}\right\} \in((C, k),|C,-1|), k \geqq-1$, if and only if $\sum n^{k+1}\left|\varepsilon_{n}\right|<\infty$.

(b) The necessity of $\sum\left|\varepsilon_{n}\right|<\infty$ follows by the argument used in part $(a)$. For the sufficiency, since $\sum a_{n}$ converges and $\mu_{n} a_{n}=o(1)$, we have

$$
\sum\left|a_{n} \varepsilon_{n}\right|=O(1) \sum\left|\varepsilon_{n}\right|<\infty \text {, and } \sum\left|\Delta\left(\mu_{n} a_{n} \varepsilon_{n}\right)\right|=O(1) \sum\left|\varepsilon_{n}\right|<\infty \text {. }
$$

(c) It is well known that (ii) is necessary and sufficient for $\varepsilon_{n} \in(|C, 0|,|C, 0|)$. By the theorem of Knopp and Lorentz referred to in Theorem 2, we find that (i) is necessary and sufficient for $\sum\left|\Delta\left(\mu_{n} a_{n} \varepsilon_{n}\right)\right|<\infty$ whenever $\sum\left|a_{n}\right|<\infty$. Hence (i) and (ii) are necessary and sufficient for $(c)$. 
If $\left\{\mu_{n}\right\}$ is bounded away from zero, then (i) implies (ii). In particular, when $\lambda_{n}=n$, we have $\left\{\varepsilon_{n}\right\} \in(|C, 0|,|C,-1|)$ if and only if $n \varepsilon_{n}=O(1)$; a result which extends a theorem of Peyerimhoff [7].

(d) Noting that

$$
a_{n}=\frac{1}{n(n+1)} \sum_{m=1}^{n} m \Delta t_{m} \quad(n \geqq 1),
$$

and again applying the Knopp-Lorentz theorem, we see that $\left\{\varepsilon_{n}\right\} \in(|C,-1|,|C, 0|)$ if and only if

$$
\sup _{k>0} \sum_{n=k}^{\infty} \frac{k\left|\varepsilon_{n}\right|}{n(n+1)}<\infty,
$$

which is equivalent to the condition in $(d)$.

This completes the proof of Theorem 5 .

In our last theorem we consider matrix transformations of $\left(C, \lambda_{n},-1\right)$ summable series $\sum a_{n}$ :

$$
A_{n}(a)=\sum a_{n k} a_{k}
$$

We give necessary and sufficient conditions for $A=\left(a_{n k}\right)$ to be regular, i.e. for $A_{n} \rightarrow s(n \rightarrow \infty)$ whenever $\sum a_{n}=s\left(C, \lambda_{n},-1\right)$. In a recently submitted note [6, Theorem 1], I have given conditions for the regularity of $A$, where $A_{n} \rightarrow s(n \rightarrow \infty)$ whenever $\sum a_{n}=s(C,-1)$. Thus, Theorem 6 generalizes this result. Since the proof of Theorem 6 is essentially the same as that in [6], $\mathrm{I}$ do no more than indicate the argument.

THEOREM 6. $A_{n}(a) \rightarrow s(n \rightarrow \infty)$ whenever $\sum a_{n}=s\left(C, \lambda_{n},-1\right)$, if and only if there is a regular series to sequence matrix $B=\left(b_{n k}\right)$ such that

$$
a_{n k}=b_{n k}+\mu_{k}\left(b_{n k}-b_{n, k+1}\right) \text {. }
$$

Proof. Let (3) hold and $\sum a_{n}=s\left(C, \lambda_{n},-1\right)$. Since $\sum a_{k}$ converges to $s$ and $B$ is regular, the $B$-transform of $\sum a_{k}$ converges to $s$. Also the matrix $\left(b_{n k}-b_{n, k+1}\right)$ takes null sequences into null sequences. Hence (3) is sufficient.

Now suppose that $A_{n}(a)$ exists for each $n$ and $A_{n}(a) \rightarrow s(n \rightarrow \infty)$ whenever

$$
\sum a_{n}=s\left(C, \lambda_{n},-1\right),
$$

i.e. by (1), whenever $t \in c$ ( $c$ being the space of convergent sequences $t=\left\{t_{n}\right\}$ with norm $\left.\|t\|=\sup \left|t_{n}\right|\right)$. If we express $a=\left\{a_{n}\right\}$ in terms of $t$ we easily find that $a_{n}=a_{n}(t)$ is a continuous linear functional on $c$. Since $\sum a_{n k} a_{k}$ converges for each $n$, it follows that $A_{n}(a)$ is a continuous linear functional on $c$. Thus, for each $n[1, \mathrm{p} .65]$,

$$
\begin{gathered}
A_{n}(a)=d_{n} \lim t_{k}+\sum_{k} d_{n k} t_{k}, \\
\left\|A_{n}\right\|=\left|d_{n}\right|+\sum_{k}\left|d_{n k}\right|, \quad \text { with } \sum_{k}\left|d_{n k}\right|<\infty .
\end{gathered}
$$


Taking $a=e^{(k)}, e_{i}^{(k)}=0, i \neq k, e_{k}^{(k)}=1$, we deduce from (4) the existence of a matrix $B$ such that (3) holds and $b_{n k}-b_{n, k+1}=d_{n k}$. Also we see that $a_{n k} \rightarrow 1(n \rightarrow \infty, k$ fixed). On applying the Banach-Steinhaus theorem, (5) yields

$$
\sup _{n} \sum_{k}\left|b_{n k}-b_{n, k+1}\right|<\infty \text {. }
$$

Taking $t=e^{(k)}$ we have $d_{n k} \rightarrow 0(n \rightarrow \infty, k$ fixed $)$, whence

$$
b_{n k} \rightarrow 1 \quad(n \rightarrow \infty, k \text { fixed }) .
$$

But (6) and (7) are the conditions for $B$ to be a regular series to sequence matrix, so the result is proved.

\section{REFERENCES}

1. S. Banach, Théorie des opérations linéaires (New York, 1955).

2. H. C. Chow, Note on convergence and summability factors, J. London Math. Soc. 29 (1954), 459-476. 1915).

3. G. H. Hardy and M. Riesz, The general theory of Dirichlet's series (Cambridge Tract No. 18, 10-16.

4. K. Knopp and G. G. Lorentz, Beiträge zur absoluten Limitierung, Archiv. der Math. 2 (1949),

5. B. Kuttner, On discontinuous Riesz means of type $n$, J. London Math. Soc. 37 (1962), 354-364.

6. I. J. Maddox, Matrix transformations of $(C,-1)$ summable series, Proc. Koninkl. Nederl. Akad. van Wetenschappen A 68 (1965), 129-132.

7. A. Peyerimhoff, Summierbarkeitsfaktoren für absolut Cesàro-summierbare Reihen, Math. $Z$. 59 (1954), 417-424.

8. W. H. Young, On the convergence of the derived series of a Fourier series, Proc. London Math. Soc. (2) 17 (1918), 195-236.

\section{THE UNIVERSITY}

\section{LANCASTER}

